\title{
Keplerian consequences of an impact on an asteroid and their relevance for a deflection demonstration mission
}

\author{
Andreas Rathke ${ }^{1}$ and Dario Izzo ${ }^{2}$ \\ ${ }^{1}$ Astrium GmbH, Dept. AED41, 88039 Friedrichshafen, Germany \\ e-mail: andreas.rathke@astrium.eads.net \\ ${ }^{2}$ European Space Agency, Advanced Concepts Team, EUI-ACT, ESTEC, Keplerlaan 1, \\ 2201 AZ Noordwijk, The Netherlands \\ e-mail: dario.izzo@esa.int
}

\begin{abstract}
We investigate upon the change of an asteroid orbit caused by an impact. We find that, given the assumption of two dimensional motion, the asteroid displacement may be described by an analytic and explicit expression that is the vectorial sum of a radial component and a component along the asteroid velocity. The new formulation bridges the gap between the study of short-term effects, using numerical methods and the analytic study of secular changes of the asteroid orbit. The relation of the method to the established formulations is described and the known results are derived as limiting cases.

The application of the new method for the performance evaluation of an asteroid deflection demonstration mission is illustrated. In such a mission the measurement of the change of the asteroid orbit by an impact will be conducted by radio-ranging to a spacecraft orbiting the deflected asteroid. Hence the measurement will primarily be sensitive to the deflection projected onto the Earth-asteroid line of sight. We discuss how the new formulation of the deflection can conveniently be employed for the estimation of the measurement accuracy and the optimal planning of a deflection demonstration mission.
\end{abstract}

Keywords. Celestial Mechanics; Space vehicles

In memoriam Floh

\section{Introduction}

One of the most promising strategies for mitigating the threat of an asteroid on collision course with the Earth is the impulsive deflection of the asteroid by a kinetic impactor. The key advantages of this method compared to deflection by applying long-duration thrust to the asteroid or its explosive destruction are simplicity and technical maturity. In particular the Deep Impact space mission [see A'Hearn et al. (2005) for an overview] has demonstrated that a high velocity impact onto a small celestial body is indeed technologically feasible.

From the astrodynamics point of view the impulsive deflection of an asteroid has already received considerable attention and can be regarded as well understood. It has been demonstrated by Carusi et al. (2002) that the most promising strategy is to apply a small velocity change to the asteroid several orbital periods before the collision with Earth or before the passage through the keyhole of a resonant return [see Valsecchi et al. (2003) for an exposition of the keyhole concept] by a tangential impact. The tangential impact leads to a change of the orbital period of the asteroid and hence to a secular change of the orbit. This secular change will accumulate over time and - even for a minuscule 
change of asteroid velocity - lead to a large accumulated miss distance. This method has meanwhile been implemented into a full end-to-end trajectory optimisation procedure that maximises the secular change of an asteroid's orbit achieved by a spacecraft departing from Earth and reaching the asteroid either by chemical or electric propulsion in Izzo (2005), Izzo et al. (2006a) and Izzo (2006b).

It is also known from the numerical study of Conway (2001) that, if the time between the deflection and the potential collision with the Earth is small, the optimal impact direction is no longer tangential to the asteroid orbit. This has recently also been verified by Kahle et al. (2006) for a deflection taking place shortly before the passage through a keyhole.

Up to now the short term regime (i.e. deflections taking place two or less orbits before the dangerous close encounter) has not been described by analytical methods. In the present paper we fill this gap by deriving an analytical description of the response of an asteroid to a small impulsive velocity change. Our result is a solution for the relative orbital dynamics between the perturbed and unperturbed orbit to linear order in the change of velocity. In its present form the solution is restricted to a velocity change in the orbital plane of the asteroid but it can be used advantageously both for short term and for long term deflections. In particular the well known secular orbital change is recovered in the limit of a long term deflection.

The study of the linearised equations of relative orbital motion has already received long-standing attention in the context of spacecraft rendezvous manoeuvres and formation flying. In this realm the relative motion is typically treated in a local-horizontal local-vertical (LHLV) coordinate system. The equations of relative motion in these coordinates are the so-called Tschauner-Hempel equations [cf. Tschauner \& Hempel (1964)], a system of three linear differential equations in the true-anomaly difference with periodic coefficients. For a circular orbit the Tschauner-Hempel equations reduce to three linear differential equations with fixed coefficients, the Clohessy-Wiltshire equations [cf. Clohessy \& Wiltshire (1960)]. If rewritten in LHLV coordinates, our solution is an explicit algebraic solution of the Tschauner-Hempel equations. For the case of a circular orbit, we establish the equivalence of our solution to the well know analytic solution of the Clohessy-Wiltshire equations.

In order to illustrate the usefulness of our solution we apply it to the performance evaluation for a space mission that aims at demonstrating the capability to deflect an asteroid. A deflection demonstration mission that is currently being studied by ESA under the name Don Quijote [cf. Carnelli et al. (2006)] is taken as the example case.

The layout of this paper is the following: We start by formulating the deflection of an asteroid in terms of the perturbation of the asteroid state vector in Sec. 2. For this formulation of the problem we first obtain the solution for the radial perturbation in Sec. 2.1 and then for the tangential perturbation in Sec. 2.2. The combined result, yielding the total deflection, is then discussed in Sec. 2.3. In Sec. 3 we apply the solution to the performance evaluation for a deflection demonstration mission. We close with a summary of our key results and give an outlook on further extensions and applications of the new method in Sec. 4.

\section{Impulsive deflection as a perturbation of the state vector}

For the present study we restrict our analysis to the case of a velocity change in the orbital plane of the asteroid. While the generalisation to out of plane deflection is straightforward it is of little practical relevance for a space mission that performs an asteroid deflection rehearsal. In the following we will determine the deflection of the 
asteroid with respect to the unperturbed orbit of the asteroid. For the unperturbed orbit we choose a description in terms of the propagation of the initial state vector $\left[\mathbf{r}_{0}, \mathbf{v}_{0}\right]$ at the time $t_{0}$ by means of the Lagrange coefficients. The radius vector at other times is then given by

$$
\mathbf{r}=F \mathbf{r}_{0}+G \mathbf{v}_{0}
$$

Here $F$ and $G$ are the Lagrange coefficients,

$$
F=1-\frac{r}{p}(1-\cos \theta) \text { and } \quad G=\frac{r r_{0}}{\sqrt{\mu p}} \sin \theta
$$

where

$$
r=\frac{p r_{0}}{r_{0}+\left(p-r_{0}\right) \cos \theta-\sqrt{p} \sigma_{0} \sin \theta}
$$

and $p$ is the orbit parameter, $\theta$ is the true anomaly difference between $\mathbf{r}_{0}$ and $\mathbf{r}, \sigma_{0} \equiv$ $\mathbf{r}_{0} \cdot \mathbf{v}_{0} / \sqrt{\mu}=r_{0} v_{0} \cos \gamma_{0} / \sqrt{\mu}, \gamma_{0}$ denotes the flight path angle at $t_{0}$ and $\mu$ is the gravitational parameter of the Sun.

If the orbit is perturbed by a velocity change $\Delta \mathbf{V}$ at the instant $t_{0}$ then the perturbation on the radius (that from now on will be called the deflection) is be given by

$$
\Delta \mathbf{r}=\Delta F \mathbf{r}_{0}+\Delta G \mathbf{v}_{0}+G \Delta \mathbf{V} .
$$

Here $\Delta F$ and $\Delta G$ denote the perturbed Lagrange coefficients.

In order to facilitate the calculation it is helpful to choose a non-orthogonal coordinate system for $\Delta \mathbf{r}$. We decompose $\Delta \mathbf{r}$ into a component along the unperturbed radius vector and a component along the unperturbed velocity,

$$
\Delta \mathbf{r}=\Delta q \mathbf{i}_{r}+\Delta s \mathbf{i}_{v},
$$

where $\mathbf{i}_{r}$ is the unit vector in radial direction and $\mathbf{i}_{v}$ is the unit vector in velocity direction. Using this system of skew axis the contravariant component of the perturbation in the radial direction is equal to the perturbation of the absolute value of the radius vector under the constraint of unperturbed difference in true anomaly $\theta$ between $\mathbf{r}_{0}$ and $\mathbf{r}$,

$$
\Delta q=\left.\Delta r\right|_{\Delta \theta=0} .
$$

Hence one can easily perturb Eq. (2.3) for the determination of $\Delta q$. A further advantage of the use of these skew axis is that the secular change of orbit will only affect the component along the velocity vector. Hence one retains the intuitive interpretation of the secular term that would for instance be lost in the LHLV coordinate system.

\subsection{The radial part}

The equation for the absolute value of the radius in terms of the initial radius, initial velocity and true-anomaly difference is given by the polar form of the equation of orbit Eq. (2.3). The change of the radius $\Delta q$ is determined to linear order by variation of parameters under the constraint that $\theta$ is unperturbed,

$$
\Delta q=\frac{r^{2}}{\sqrt{p} r_{0}} \sin \theta \Delta \sigma-\frac{r^{2} \cos \theta}{p r_{0}} \Delta p+\frac{r^{2} \sigma_{0}}{2 p^{3 / 2} r_{0}} \sin \theta \Delta p+\frac{r}{p} \Delta p .
$$

The variations of $p$ and $\sigma_{0}$ by a $\boldsymbol{\Delta} \mathbf{V}$ transfer are in turn given by

$$
\begin{aligned}
& \Delta p=2 \sqrt{\frac{p}{\mu}}\left|\mathbf{r}_{0} \times \Delta \mathbf{V}\right|=2 \sqrt{\frac{p}{\mu}} r_{0} \Delta V \sin \phi, \\
& \Delta \sigma=\frac{\mathbf{r}_{0} \cdot \Delta \mathbf{V}}{\sqrt{\mu}}=\frac{r_{0} \Delta V}{\sqrt{\mu}} \cos \phi,
\end{aligned}
$$


where $\phi$ is the angle between $\mathbf{r}_{0}$ and $\boldsymbol{\Delta} \mathbf{V}$. Inserting these expressions we arrive at

$$
\begin{aligned}
\Delta q=r & \frac{2 r_{0}}{\sqrt{\mu p}} \sin \phi \Delta V \\
& \quad+r^{2} \frac{r_{0} v_{0}}{\mu p}\left[\sin \gamma_{0} \cos \phi \sin \theta-2 \sin \gamma_{0} \sin \phi \cos \theta+\cos \gamma_{0} \sin \phi \sin \theta\right] \Delta V
\end{aligned}
$$

where we have used $\sqrt{\mu p}=r_{0} v_{0} \sin \gamma_{0}$ and $\mathbf{r}_{0} \cdot \mathbf{v}_{0}=r_{0} v_{0} \cos \gamma_{0}$. In Eq. (2.10) the terms with $\sin \phi$ correspond to a change of orbital angular momentum while the term proportional to $\cos \phi$ corresponds to a change of orbital energy.

\subsection{The tangential part}

In order to obtain the tangential part of the deflection one has to consider the full variation of the state vector. Singling out the components along the velocity vector $\mathbf{v}$ one obtains

$$
\begin{aligned}
\Delta s \mathbf{i}_{v}= & \mathbf{v} \\
- & -\frac{3 a}{\mu}\left[t-t_{0}\right]\left(\mathbf{v}_{0} \cdot \Delta \mathbf{V}\right)-\frac{3 a^{2}}{\mu^{3 / 2}}\left(\sigma-\sigma_{0}\right)\left(\mathbf{v}_{0} \cdot \Delta \mathbf{V}\right) \\
- & \frac{3 a^{2} r \sigma_{0}}{\mu^{3 / 2} p}(1-\cos \theta)\left(\mathbf{v}_{0} \cdot \Delta \mathbf{V}\right)+\frac{a r_{0} r}{\mu^{3 / 2} p}\left(\sigma-\sigma_{0}\right)(1-\cos \theta)\left(\mathbf{v}_{0} \cdot \Delta \mathbf{V}\right) \\
& +\frac{3 a^{2} r}{\mu^{3 / 2} \sqrt{p}} \sin \theta\left(\mathbf{v}_{0} \cdot \Delta \mathbf{V}\right)+\frac{r_{0}^{2} r^{2}}{(\mu p)^{3 / 2}}(1-\cos \theta) \sin \theta\left(\mathbf{v}_{0} \cdot \Delta \mathbf{V}\right) \\
- & \frac{r_{0} r}{\mu p}(1-\cos \theta)\left(\mathbf{r}_{0} \cdot \Delta \mathbf{V}\right)-\frac{r_{0} r^{2} v_{0}}{(\mu p)^{3 / 2}}(1-\cos \theta) \sin \left(\theta-\gamma_{0}\right)\left(\mathbf{r}_{0} \cdot \Delta \mathbf{V}\right) \\
& \left.-\frac{r^{2} r_{0}}{\mu p} \sin \theta \sin (\theta-\phi) \Delta V\right\}
\end{aligned}
$$

where we have introduced $\sigma=\mathbf{r} \cdot \mathbf{v} / \sqrt{\mu}$ and $a$ denotes the semimajor axis.

Several of the terms of Eq. 2.11 have a direct interpretation. Most notably the first term proportional to the time after impact, $t-t_{0}$, is the only secular perturbation of the orbit. This term had already been obtained in Izzo (2005) and compared in magnitude to the other terms for different orbits and times. The next three terms are effects of the eccentricity of the unperturbed orbit and vanish for a circular orbit. Again all terms with the exception of the last term can be associated with either a change of orbital energy (term 1-6) or angular momentum (term 7 and 8). The last term is the projection of the third term in Eq. (2.4).

\subsection{The total deflection}

The total deflection is obtained by inserting Eqs. (2.10) and (2.11) into Eq. (2.5). The total deflection is a an exact solution to linearised equations of motion for the relative motion between the perturbed and unperturbed orbit. Higher order terms will only lead to corrections of order $O\left(\Delta V / v_{0}\right)^{2}$. For the case of a real asteroid deflection $\Delta V$ is foreseen to be of the order $10^{-5} \mathrm{~m} / \mathrm{s}$ while the orbital velocity will be of the order $10 \mathrm{~km} / \mathrm{s}$. Hence corrections to Eq. (2.5) will be suppressed by a factor $10^{-9}$ and higher order terms can be neglected without introducing significant errors. $\dagger$

$\dagger$ The situation is analogous to that of spacecraft rendezvous in Earth orbit. Also there the relative motion is usually only treated to linear order for all practical applications. 
While the new expression is fully analytical and explicit, it depends on both the true anomaly difference, $\theta$, and time after the impulsive velocity change, $t-t_{0}$. Hence the application of the solution requires solving Kepler's equation for the unperturbed orbit.

In conclusion, the new deflection equation gives a full description of the change of a Keplerian orbit after an impulsive velocity change. After transformation into a LHLV coordinate system the solution becomes an analytic and explicit solution of the TschaunerHempel equations for the relative dynamics on elliptic orbits.

Indeed similar formulations in terms of perturbation matrices have already been obtained previously [see e.g. Battin (1994) p. 463ff. for an introduction to the topic]. The new formulation has however a significant advantage over the known formulations. In order compute the deflection in terms of perturbation matrices one has to numerically solve the variational equation for the secular part of the deflection. In our new formulation the secular term is given explicit and analytic in terms of the time after impact and the non-secular terms are given explicit and analytic in terms of the true anomaly difference.

Our new formulation encompasses the established treatments of long-term impulsive asteroid deflection in Carusi et al. (2002) and Izzo (2006b). These results of these studies are recovered in the limit of long times after the deflective impact. The particular power of the new method lies however in the regime of mid-term deflection, that is the case where the deflection has to be achieved a few orbital periods before the collision with the Earth. On the one hand, in this regime the radial term is not yet negligible and needs to be taken into account. On the other hand the propagation time after deflection is already so long that an analytical treatment of the secular term has significant advantages over numerical schemes.

It is also straightforward to show that our solution reduces to the solution of the Clohessy-Wiltshire equations [cf. Clohessy \& Wiltshire (1960)] for the case of a circular orbit because for this case the LHLV coordinate system and the radial-tangential coordinate system coincide. The equivalence of the two solutions is readily established by noting that for a circular orbit we have $\sigma=\sigma_{0} \equiv 0$ and exploiting the simplifications of the Lagrange coefficients that arise for circular orbits

$$
F_{t}=-G, \text { and } G_{t}=F,
$$

where $F_{t}$ and $G_{t}$ denote the Lagrange coefficients for the equation for the velocity vector $\mathbf{v}=F_{t} \mathbf{r}_{0}+G_{t} \mathbf{v}_{0}$. From Eq. (2.12) we have the relation

$$
(1-F) \mathbf{r}+G \mathbf{v}=-(1-F) \mathbf{r}_{0}+G \mathbf{v}_{0},
$$

by which we can easily transform Eq. (2.11) into the tangential component of the solution of the Clohessy-Wiltshire equations for an impulsive orbit change.

\section{Application to deflection demonstration missions}

The perturbative solution is particularly suited for the assessment and optimisation of the performance of a deflection demonstration space mission. The basic principle of a deflection demonstration mission is to impact an asteroid that poses no threat to the Earth and measure the achieved deflection. The measurement of the achieved deflection is of crucial importance because it yields a measurement of the momentum carried away by ejecta. It is expected that the momentum transfer to the asteroid from the shedding of ejecta will considerably exceed the momentum transfered by the impact itself. Hence, the determination of the momentum transfer caused by the impact achieved by measuring the orbital change of the asteroid gives more reliable estimates for future deflection missions. 
A deflection demonstration that follows this principle is currently being studied by ESA under the name Don Quijote [see Carnelli et al. (2006) for an overview]. The Don Quijote mission is envisaged to comprise two spacecraft, the Orbiter and the Impactor. The Orbiter will be launched first. It will travel to the asteroid and conduct a precision determination of the asteroid orbit and carry out a precision measurement of its mass, gravity field, topography, rotational motion and composition. After the successful arrival of the Orbiter at the asteroid the Impactor will be launched from Earth. It will travel to the asteroid and collide with it in a way to achieve the largest possible change of the orbital energy of the asteroid. The minimum goal is to change the semimajor axis of the asteroid orbit by $100 \mathrm{~m}$. The Orbiter will observe the collision of the Impactor from a safe position and analyse the ejecta. Then it will return to closer proximity of the asteroid and conduct a second orbit determination campaign in order to measure the deflection that has been achieved.

In a deflection demonstration mission the smallness of the orbital changes makes it necessary to measure it via radio-tracking of a transponder orbiting the asteroid (or placed on it). Consequently the mission layout has to take into account the peculiarities of radio-tracking measurements. In particular the measurement will be sensitive primarily to special components of the deflection in the geocentric frame because the measurement will be carried out from a groundstation on Earth.

The methods of choice for the deflection measurement are ranging and differential very large baseline interferometry $(\Delta \mathrm{VLBI})$ [see Thornton \& Border (2000) for a description of these techniques and their performance]. In particular Doppler tracking can immediately be excluded as a suitable method because the velocity change of the asteroid will be in the order of 1 to $10 \times 10^{-5} \mathrm{~m} / \mathrm{s}$ whereas the absolute accuracy of present-day Doppler spacecraft tracking is limited to approximately $10^{-3} \mathrm{~m} / \mathrm{s}$ post-processing accuracy.

Radio ranging, in which the runtime of a signal to the spacecraft and back to the ground station is measured, is sensitive primarily to the distance change between the asteroid and the Earth. Hence this method will be sensitive to the deflection along the line-of-sight between the asteroid and the Earth. As a rule of thumb we can expect a post processing accuracy for the ranging of about $10 \mathrm{~m}$, which will be dominated by the uncertainty in the position of the asteroid centre of mass which respect to the Orbiter.

$\triangle$ VLBI measures the angular position of the Orbiter on the sky relative to a known astronomical radio source such as a quasar by triangulation with two ground stations. Hence it is mainly sensitive to the asteroid's change in angular position, i.e. the component of the deflection orthogonal to the Earth line of sight. With present-day equipment a post-processing accuracy of $50 \mathrm{nrad}$ is achieved. For upcoming missions with new equipment we can however assume a post-processing accuracy of $5 \mathrm{nrad}$, which we will assume in our analysis [cf. Thornton \& Border (2000) p. 65]. As a consequence, the deflection needs to be assessed in terms of the projections onto the measurable quantities in the geocentric frame.

While the above performance values give a rule of thumb, the precise expected performance for the orbit determination of the asteroid from the radio measurements can only be determined from a complete simulation. Important parameters, that will influence the measurement performance, are the Yarkowsky effect on the asteroid, and the correct estimation of it, the determination of the asteroid centre of mass position with respect to the Orbiter, the knowledge of non-gravitational disturbances on the Orbiter, and effects on the radio signal propagation.

Hence a determination of the measurement performance from simple astrodynamical considerations remains elusive. Nevertheless the orbital change of the asteroid in the measurement frame provides an important figure of merit. Such a figure of merit is 
Table 1. Asteroid and Impact parameters for the two Don-Quijote strawman targets.

\begin{tabular}{lcc}
\hline Asteroid & $(10302) 1989 \mathrm{ML}$ & $2002 \mathrm{AT}_{4}$ \\
\hline \multicolumn{3}{c}{ Asteroid parameters } \\
a (AU) & 1.27 & 1.87 \\
perihelion $(\mathrm{AU})$ & 1.10 & 1.03 \\
$\mathrm{e}$ & 0.14 & 0.45 \\
$\mathrm{P}$ (days) & 524 & 931 \\
& Impact parameters & \\
Impact date & 6 Feb. 2018 & $9 \mathrm{April} 2017$ \\
$\phi(\mathrm{deg})$ & -29 & -70 \\
$\Delta V(\mathrm{~m} / \mathrm{s})$ & $10^{-5}$ & $6 \times 10^{-5}$ \\
$\Delta a(\mathrm{~m})$ & 96.4 & 2232 \\
\hline
\end{tabular}

in particular necessary for the design a suitable Impactor trajectory. Due to the large number of possible trajectories, it is not feasible, in practice, to evaluate the suitability of each of them by a full radio-tracking simulation. The simple geometrical quantities provide a reliable criterion if a particular Impactor trajectory is suitable to achieve a measurable deflection. The projections of the deflection onto the Earth line of sight $x_{\|}$ and orthogonal to it $x_{\perp}$ are given by

$$
\begin{aligned}
x_{\|} & =\Delta q\left(\mathbf{i}_{r} \cdot \mathbf{i}_{\oplus \mathrm{A}}\right)+\Delta s\left(\mathbf{i}_{v} \cdot \mathbf{i}_{\oplus \mathrm{A}}\right), \\
x_{\perp} & =\Delta q\left|\mathbf{i}_{r} \times \mathbf{i}_{\oplus \mathrm{A}}\right|+\Delta s\left|\mathbf{i}_{v} \times \mathbf{i}_{\oplus \mathrm{A}}\right|,
\end{aligned}
$$

where $\mathbf{i}_{r} \equiv \mathbf{r} / r, \mathbf{i}_{v} \equiv \mathbf{v} / v$ and $\mathbf{i}_{\oplus \mathrm{A}}$ is the unit vector along the Earth asteroid line of sight.

In a deflection demonstration mission the measurement time after the deflection will typically be limited in order to limit the overall mission duration. For example, for the Don Quijote study ESA aims at completing the deflection measurement within 1/2 year after the impact. Noting that the period of a Near Earth Asteroid will be one year or even significantly more it is immediately obvious that such a measurement time lies in the last-minute regime of asteroid deflection. Hence it becomes necessary to build the performance meter for the deflection on the full (albeit linearised) orbital change taking into account both, secular and non-secular terms.

For the Don Quijote study ESA chose not to aim at a deflection that is optimally measurable but instead has chosen a scenario that is close to a real case of long-term deflection: An Impactor trajectory is considered optimal if it achieves a change of semimajor axis of $\Delta a=100 \mathrm{~m}$ at minimal mission cost and complexity. This corresponds to an optimisation of the secular deflection. In order to determine if this is a viable strategy it has to be considered if the trajectories optimised in this way lead to a deflection that is measurable in the geocentric frame.

We approach this problem by considering trajectories to both Don Quijote targets that have been optimised for a change of semimajor axis and evaluate the measurable deflection for them. The trajectories that we consider have been obtained in the framework of an ongoing industrial phase A study of the Don Quijote mission by the company Deimos Space. They assume a launch with the Russian Dnepr launcher and Earth escape with a LISA-Pathfinder propulsion module. Furthermore the Sun-asteroid-Impactor angle is limited to $70 \mathrm{deg}$ during the terminal approach to ensure sufficient illumination for visual terminal navigation.

Some key parameters of the target asteroid orbit and the parameters characterising the impact are given in Table 1 . The achievable velocity increments $\Delta V$ are based on rough mass estimates for the asteroids and the assumption of a totally inelastic collision. 
Figures 1 to 4 display the result of our analysis. Figure 1 displays the deflection for the asteroid (10302) 1989 ML. The maximal orbital change along the direction of the Earth line-of-sight reaches approximately $550 \mathrm{~m}$. At the end of the measurement period the measurable deflection rapidly decays because the Earth has 'overtaken' the asteroid and the tangential and and radial deflection partially compensate each other in the projection along the Earth line-of-sight. The maximal measurable deflection is reached near the opposition of the asteroid. Briefly before that the Earth line-of-sight is aligned with the vector of the deflection. At this instance the measurable deflection and the total deflection coincide. The change of the relative position of Earth causes a strong modulation of the measurable deflection component. Clearly the secular term is neither representative for the total deflection nor for the tangential deflection. Still in this scenario, that has been optimised for the secular term, the measurable deflection is one order of magnitude larger than the expected ranging accuracy in the second half of the measurement period. This shows that a reliable measurement of the deflection is feasible in this scenario. The angular deflection of 1989 ML seen from the Earth is displayed in Figure 2. The angular deflection remains below the capabilities of $\triangle \mathrm{VLBI}$ for the first 300 days after the impact. A precise measurement of the deflection is not possible with this method.

Figure 3 shows the deflection achieved for $2002 \mathrm{AT}_{4}$. The deflection is nearly one order of magnitude larger than for $1989 \mathrm{ML}$. For this asteroid the observation period comprises less than half of orbital period and is hence truly in the 'last minute' regime of deflection. Also for this object the secular term is not representative of the total deflection or the tangential deflection. Despite of the much larger deflection, the angular deflection is even smaller than for $1989 \mathrm{ML}$. The reasons for this are the larger geocentric distance of the asteroid during the measurement period and the higher eccentricity of the orbit by which the tangential deflection is quite well aligned with the Earth direction for a considerable part of the orbit after the impact.

The two examples show that for the limited measurement duration of a deflection demonstration the secular and non-secular contributions to the deflection are equally important. The modulation of the measureable deflection by the relative motion of the Earth has a strong influence on the magnitude of the measureable deflection. In particular, for certain Earth-asteroid configurations the contributions of the radial and tangential deflection to the apparent deflection can compensate each other. The optimal measurement situation is only achieved if the apparent deflection is optimised.

The two examples also demonstrate that the secular term (i.e. the semimajor axis change) is by no means representative of the measurable deflection in a deflection demonstration mission.

\section{Conclusions}

In the present study we have analysed the orbital change of a Keplerian orbit by an impulsive velocity change in the orbital plane. A solution for the relative dynamics with respect to the unperturbed orbit was obtained up to linear order. The solution is algebraic, analytic and explicit albeit depending on both the time after the velocity change and the relative true anomaly difference to the point of velocity change. It is well suited for an implementation into a delfection optimisation procedure.

The solution was formulated in a non-orthogonal coordinate system with its principle axis along the unperturbed radius vector and the unperturbed velocity. This coordinate system is advantageous because the secular change of the orbit is oriented tangentially to the orbit and hence secular changes are limited to one of the coordinate vectors. From the 


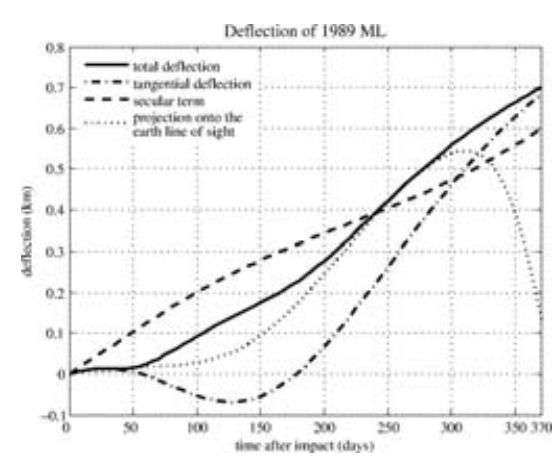

Figure 1. Deflection of $1989 \mathrm{ML}$ and components thereof

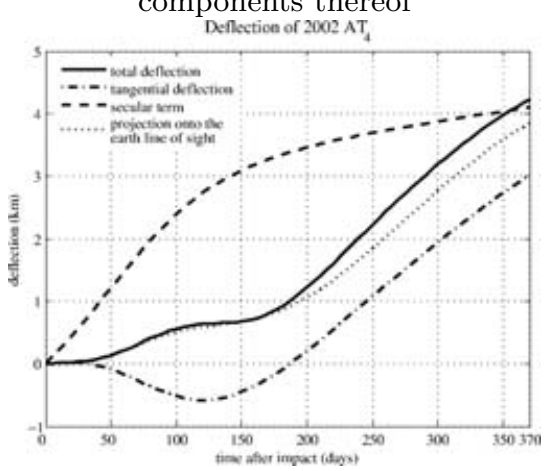

Figure 3. Deflection of $2002 \mathrm{AT}_{4}$ and components thereof

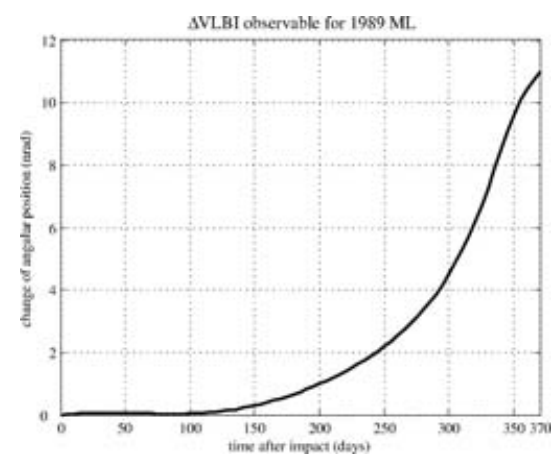

Figure 2. Angular deflection of 1989 ML as seen from Earth $\Delta$ VLBI observable for $2002 \mathrm{AT}_{4}$

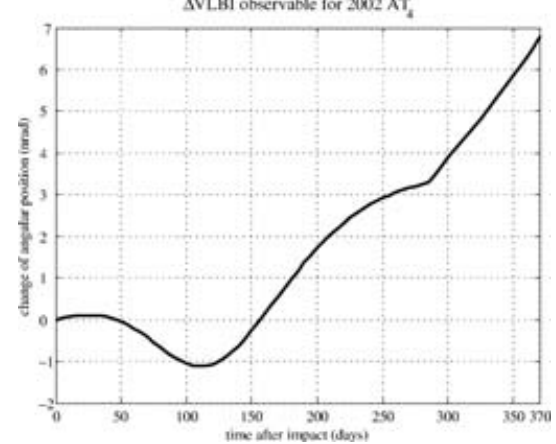

Figure 4. Angular deflection of $2002 \mathrm{AT}_{4}$ as seen from Earth

new solution an explicit analytic solution of the Tschauner-Hempel equations is easily obtained by projecting the solution into the LHLV coordinate system.

As an application of the new solution we studied the orbital deflection of an asteroid after the impact of a spacecraft. For a velocity change which is small compared to the orbital velocity of the asteroid the solution describes accurately the relative dynamics of the perturbed orbit compared to the unperturbed one. The new formulation is in particular relevant for the description of the perturbation in the first few orbital revolutions after the impact. For long times after the impact the secular perturbation of orbit becomes dominant because it exhibits a linear growth with time whereas all the other terms show a periodicity shorter or equal to one orbital period. A scenario in which the non-secular terms are still important is that of mid-term deflection in which a collision with Earth in the next few orbital periods needs to be mitigated. The effect of the non-secular terms is even more significant for a deflection demonstration mission because for practical reasons one will want to measure the achieved deflection on a timescale that will hardly exceed one year.

The equal importance of all contributions in the deflection measurement of a demonstration mission has been illustrated using two example cases which are based on the Don Quijote study currently being carried out by ESA. For each of the two strawman targets of the Don Quijote mission, $1989 \mathrm{ML}$ and $2002 \mathrm{AT}_{4}$, a trajectory optimised for a maximal secular change of the asteroid orbit was considered. It was found that the secular term is not representative for the perturbation of the asteroid orbit that is achieved. The reasons are two-fold: Firstly, the non-secular terms are of the same magnitude. Sec- 
ondly the measurement of the deflection is primarily sensitive to the deflection along the Earth-asteroid line of sight. This singles out a projection of the total deflection that does not coincide with the deflection tangential to the asteroid orbit. While the two effects do not exclude the measurement of the deflection in the example cases, they delay the reliable detectability of the deflection - in the case of 1989 ML by several month.

In conclusion, a deflection demonstration mission can either be designed as closely as possible to a real case deflection by optimising the secular change of the asteroid orbit or it can be optimised to achieve a large measurable deflection by maximising the deflection along the Earth-asteroid line-of-sight. Choosing the latter option has the potential of considerable cost savings because a smaller velocity change of the asteroid orbit will be sufficient to verify the ability of carrying out a real-case deflection.

Trajectory planning that optimises the measurable deflection is readily accomplished by implementation of the new analytic deflection description into an optimiser. Due to the analyticity of the new expressions the optimisation procedure will only marginally exceed the computational requirements of a trajectory optimisation that maximises the secular deflection. An implementation of an optimisation procedure for the measurable deflection in a deflection demonstration mission is currently underway.

\section{Acknowledgements}

The authors are grateful to Juan L. Cano from Deimos Space for providing the example Impactor transfer trajectories and to Diego Escorial Olmos for useful discussions on the ranging measurements.

\section{References}

A'Hearn, M.F., et al. 2005, Science 310, 258

Battin, R.H. 1994, An Introduction to the Mathematics and Methods of Astrodynamics AIAA Education Series (AIAA, Washington, DC)

Carnelli, I., Galvez, A. \& Izzo, D. 2006 "Don Quijote: A NEO deflection precursor mission" submitted to NASA Workshop: Near-Earth Object Detection, Characterization, and Threat Mitigation, http://www.esa.int/gsp/ACT/doc/ACT-RPR-4200-ICNASANEOWS-DonQuijote.pdf

Carusi, A., Valsecchi, G.B., D'Abramo, G. \& Boattini, A. 2002, Icarus 159, 417

Clohessy, W.H. \& Wiltshire, R.S. 1960, Journal of the Aerospace Sciences 27, 653

Conway, B.A. 2001, Journal of Guidance, Control, and Dynamics 24, 1035

Izzo D. 2005, in: Proceedings of the 2005 AAS/AIAA Space Flight Mechanics Conference (Univelt Inc.), Paper AAS 05-150, Vol. 121, p. 611

Izzo D., Bourdoux A., Walker R. \& Ongaro F. 2006, Acta Astronautica 59, 294

Izzo, D., "Optimisation of interplanetary trajectories for impulsive and continuous asteroid deflection," 2006, to appear in Journal of Guidance Control and Dynamics

Kahle, R., Hahn, G. \& Kührt, E. 2006, Icarus 182, 482

Thornton, C.L. \& Border, J.S. 2000, Radiometric tracking techniques for deep-space navigation Deep-space communications and navigation series 00-11, Jet Propulsion Laboratory, http://descanso.jpl.nasa.gov/Monograph/series1/Descanso1_all.pdf

Tschauner, J. \& Hempel, P. 1965, Astronautica Acta 11, 104

Valsecchi, G.B., Milani, A., Gronchi, G.F. \& Chesley, S.R. 2003, Astron Astrophys., 408, 1179 